\title{
Teaching Surgery to Medical Students
}

\author{
W. Brian Sweeney, M.D. ${ }^{1}$ \\ ${ }^{1}$ Department of Surgery, University of Massachusetts Medical School, \\ Worcester, Massachusetts \\ Clin Colon Rectal Surg 2012;25:127-133.
}

\begin{abstract}
Address for correspondence and reprint requests W. Brian Sweeney, M.D., Department of Surgery, University of Massachusetts Medical School, 55 Lake Ave., Worcester, MA 01655

(e-mail: william.sweeney@umassmemorial.org).
\end{abstract}

Teaching the discipline of surgery to medical students depends on the practicing clinical surgeon assuming the role of educator. The amount of surgical knowledge to impart and technical skill to train are ever increasing as the duration of time students spend on surgical rotations is decreasing. The intent of this article is to assist those surgeons with minimal formal training in educational methods to enhance their teaching skills. Traditional large-group lecture, small-group sessions, and mentoring are reviewed with special emphasis on medical simulation.
Objectives: On completion of this article, the reader should be able summarize options for teaching medical students depending on the size of the group and incorporate into his or her teaching several types of strategies, including medical simulation.

The education of future physicians in the United States includes a period of time, typically 4 years, called medical school. Traditionally, the first 2 years of medical school are utilized to teach the basic sciences and the last 2 years are for clinical training. In this model, students are formally taught the discipline of surgery during the third year over a period ranging from 6 weeks in some schools to up to 12 weeks in others. It is very clear that given the tremendous increase in medical knowledge, surgical technology, and intricate operative procedures, teaching surgery to medical students during this relatively short exposure has become an immense challenge. To meet the challenge, surgical educators must (1) clearly establish realistic learning objectives for the student on surgical rotations, (2) use traditional teaching methods proven to be effective, and (3) employ new modalities where appropriate. Most of all, given the impossibility of providing for an all-encompassing curriculum, surgeons must demonstrate and initiate the process of, desire for, and skills required for lifelong learning.

The responsibility of teaching surgery to medical students rests primarily on busy clinical surgeons possessing little or no formal training on educational methods and principles. We often teach how we were taught. In addition, surgeon educators range from highly published professors of surgery in prestigious academic institutions to the rural clinical surgeon with a wealth of practical experience and "clinical pearls" not found in standard surgical texts. The intent of this chapter is to review current educational methods and modalities, to encourage surgeons to reflect on their individual teaching style, and to present teaching options that can be incorporated into their particular academic surgical practice. As the art of surgery requires technical skill acquisition, simulation-based training strategies will be highlighted.

\section{Learners}

The students we are privileged to educate have excelled in their undergraduate education-maintaining an average grade point average (GPA) of 3.67. ${ }^{1}$ In addition, they have spent many hours in service to others in society, participated in numerous extracurricular activities, and often engaged in scientific research projects. Today's medical student has access to and is adept in the use of technology for learning. Educators can make use of this technologic ability as they attempt to impart knowledge and technical skills to the student. Traditional educational approaches of providing information in lecture format (passive learning) can be creatively replaced with methods encouraging student interaction with the learning environment (active learning). Medical students should be encouraged to begin taking some degree of control over their own learning by meeting personal objectives and choosing individual learning styles (selfdirected learning). The assistance these students received early on in their training can be gradually reduced allowing
Issue Theme Surgical Education in Colorectal Surgery; Guest Editor, Judith L. Trudel, M.D.
Copyright (c) 2012 by Thieme Medical Publishers, Inc., 333 Seventh Avenue, New York, NY 10001, USA. Tel: +1(212) 584-4662.
DOI http://dx.doi.org/ $10.1055 / \mathrm{s}-0032-1322525$. ISSN 1531-0043. 
more personal responsibility for their own learning (scaffolding). ${ }^{2}$ Eventually, the student and then the physician will be in charge of choosing the "what" or content and the "how" or method for their lifelong learning.

Surgeons teach in a variety of settings. The setting typically influences the size of the learner group and vice versa. Certain teaching options are then available depending on the size of the group. Three groups can be simply and arbitrarily defined based on the number of students: a mentor group (1-3 students), a small group (4-25 students), and a large group ( $>25$ students). The mentor group is appropriate for teaching in the clinic/office, bedside/rounds, operating room, procedure room (i.e., endoscopy suite). Small-group teaching occurs in small classrooms, simulation laboratories, and informal settings (cafeteria, faculty homes, off-campus venues). The large group meets in the traditional lecture hall or amphitheater.

\section{Large-Group Teaching}

The classic method for medical education today continues to be large groups of students in large classrooms. Although there is typically little or no interaction, surgical knowledge can in fact be transferred to students with the benefit of maximally using faculty time. The large-group lecture will undoubtedly be in the student's future as continued medical education occurs in vast convention centers with one or a panel of educators teaching hundreds of learners.

The large-group setting, though not optimal for technical skills and problem-solving skills training, can certainly be effective for the simple transfer of information from the surgeon educator to the medical student. There are techniques any surgeon (regardless of the lack of formal educational training) can utilize to enhance the development and delivery of his or her lecture. Helpful terms to remember when developing the content of the lecture include objectives, relevance, reinforcement, and summary. The objectives must be specific, clearly stated at the start of the lecture, and realistic for the time allotted. ${ }^{2,3}$ Answer this question: What do I expect the student to learn when my presentation is over? To pique student interest, it is also desirable early on to describe the relevance of your topic. As typical student attention span begins to decrease after 15 minutes, it is therefore important to provide brief reinforcement every 10 to 15 minutes. ${ }^{4}$ Finally, leaving the students with a practical summary can solidify the information transfer.

As an example consider a 1-hour lecture on basic proctology to a group of 100 third-year medical students. The stated objectives (what you will learn) could be: At the end of this presentation, you will (1) be able to recognize 10 common anorectal conditions affecting patients, (2) be able to state how the patients present clinically, and (3) understand the pathophysiologic basis for the symptoms produced. The relevance (why you should learn) could be pointed out as follows: The time devoted to proctology during medical school training is very brief yet the chances are great that most physicians across medical specialties will encounter these patients. Reinforcement of the information presented about common anorectal conditions can be readily provided by using operative or office photographs interspersed at intervals throughout the presentation. The summary would then emphasize that in most cases of anorectal pathology the clinician can easily diagnose a particular condition by simply listening to the presenting symptoms, remembering basic anorectal anatomy and physiology, and identifying classic findings on examination.

The goal for delivering an effective large group lecture is engagement of the audience. Techniques to engage your students can include using humor, asking students questions by using their names if possible, polling the audience by asking for a show of hands, roaming around the lecture hall, and maintaining eye contact with the students and not the projection screen. Most important in the delivery of your presentation is the demonstration of enthusiasm for your topic!

In short, the large-group lecture format is here to stay given its economic use of the educator's time. However, there are options available using Internet sites that provide the desired transfer of basic surgical knowledge while allowing for the replacement of the lecture with more active learning methods. MedU, New England Journal of Medicine Videos in Clinical Medicine, Medline Plus Surgical Procedures, and Procedures Consult are example of such sources. ${ }^{5-8}$ At the University of Massachusetts Medical School, we have begun this process of replacing traditional surgical lectures with the web-based surgical modules found in MedU called WISEMD (Web Instruction for Surgical Education). This has freed up time for increased faculty mentoring and small-group simulation sessions.

\section{Small-Group Learning}

Conducting small-group learning sessions often requires more faculty-and more faculty time-in an era when clinically active surgeons already have many demands. Nevertheless, there are benefits inherent in this method that cannot be achieved in a large-group setting. Although formal lectures can be delivered to a small group of students, more interactive options exist. Examples of small-group sessions include casebased or problem-based discussions, journal club, assigned topics, and simulation-based training. Simulation-based training requires some type of laboratory, but the other types of small-group sessions can occur in nontraditional educational settings. Holding a journal club, case discussion, or topic presentation at the faculty member's home, at a meeting room, or other off-campus venues can be very effective for stimulating student interest.

Case-based or problem-based discussions are ideal for small-group teaching. They are simple to create, structured, and easily conducted as they reflect the approach the clinician takes during a patient encounter. A surgical case or problem that is felt to be important is chosen by the educator. This can be a real case, a patient the surgeon has previously encountered, or one that the student is currently following, for example, a 50-year-old woman with crampy abdominal pain, nausea, and vomiting. A problem-based discussion typically begins with a problem or pathologic condition such as large bowel obstruction. All students in the group 
are expected to participate, encouraged to collaborate with others in the group, and made to feel comfortable that involvement will not be ridiculed. The job of the surgeon educator then is to facilitate the discussion rather than merely transferring knowledge. This is accomplished by posing questions, providing information when needed, ensuring all group members participate, and directing the case from presenting symptoms through ultimate management options. The students as a group are expected to obtain an appropriate history asking pertinent questions, describe the physical examination and reasons for the findings, order proper diagnostic tests and rationale for use, generate a reasonable differential diagnosis, and formulate a management plan. Pertinent radiographic images, laboratory displays, patient images, and operative photos may be incorporated into the presentation as indicated and as available.

The case-based/problem-based approach teaches and demonstrates cognitive skills such as data analysis, reasoning, and problem solving. The knowledge learned from passive methods such as textbooks, lectures, and Internet sources is now applied to the diagnosis and management of the problem presented. Finally, this method encourages the interaction with colleagues that ultimately is required for the practice of medicine.

Journal clubs, often used as learning tools for residents, are of value for the medical student as well. The learner group must be of small size and can consist of only medical students or even better-a combination of students and residents. Key articles are selected by the surgeon, read by all participants but assigned to specific learners who lead the discussion. The purpose of a journal club is not only the transfer of valuable state-of-the-art medical knowledge, but also the critical appraisal of study design and quality of medical literature. Journal clubs are well suited for informal settings such as a faculty member's home.
An assigned topic presentation refers to the educator requesting individual students to present to the small group a 10-minute review of a relevant topic. The student can decide which topic to present based on recent clinical experience, or alternatively have a topic assigned by the educator. In addition, respecting the learner's limited attention/retention span, the 10-minute limit allows for questions and faculty comments. This type of exercise can inspire the student to initiate, conduct, and submit research presentations to local, regional, and even national medical meetings.

\section{Simulation-Based Training}

Simulation-based training is an effective modality to teach both technical skills and nontechnical skills in a safe, nonthreatening environment. Simulation for training is widely accepted in the military (flight simulation) and in athletics (golf simulators); it is becoming increasingly implemented for medical training. Medical simulation is the representation of a real medical event by a created model for the purpose of education and/or evaluation. The medical event could be an encounter, a situation, or a procedure. The created model is the simulation.

Technical simulation is designed for technical or procedural skill training and can utilize task trainers, partial- or full-body manikins, live or cadaveric animals, human cadavers, or virtual reality computer systems (-Fig. 1). Nontechnical simulation is intended for communication, knowledge, and decision-making skills and can occur through role play, mock board examinations, and interaction with standardized patients (actors). Immersion simulation uses a combination of modalities teaching both technical skills along with nontechnical skills.

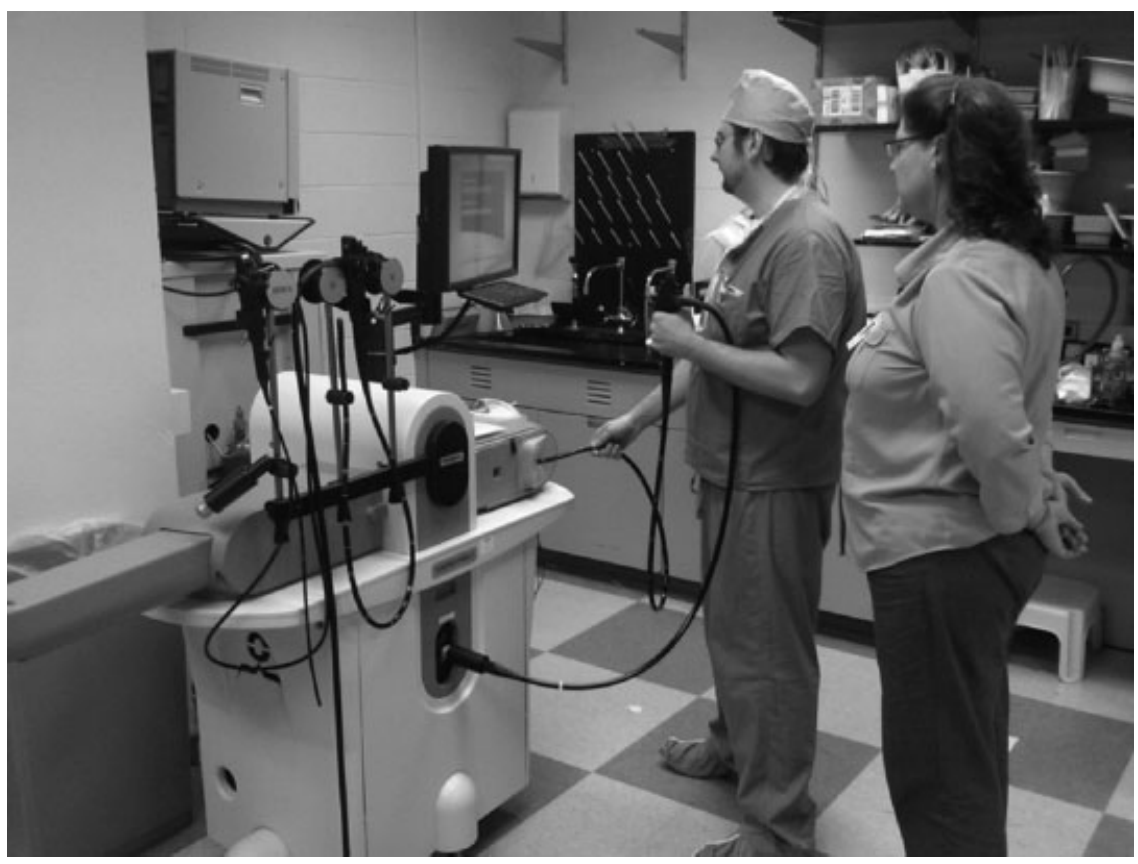

Figure 1 High-fidelity, virtual reality endoscopy simulator (Simbionix ${ }^{\mathrm{TM}}$, Cleveland, $\mathrm{OH}$ ). 
Simulated medical events can be categorized as encounters, situations, or procedures. An encounter could be the breaking of bad news to a patient or family member and the simulation would be standardized patients in a mock emergency waiting room. An example of a situation could be a cardiac arrest simulated using a high-fidelity manikin. Surgeon educators have a particular responsibility to teach technical and procedural skills. For medical students, this can be suturing and knot tying using a suitable task trainer (-Fig. 2).

Medical simulation may be used for education and also for evaluation. As an educational tool, simulation can increase the learner's knowledge base, improve decision making, teach teamwork, and develop psychomotor skills. As an evaluation tool, simulation seeks to ensure some degree of competency in the learner. Ultimately, elevated competence translates into decreased medical errors, improved patient safety, and reduced health care costs. This is the argument for integration of simulation into medical training.

Once convinced that simulation plays a role in medical education, the educator must decide how to integrate this tool into his or her particular setting. Five questions should be addressed: Who are you teaching? What are you teaching? To what level of competency are you teaching? Can simulation play a role? If so, what level of fidelity is required? As an example, consider teaching third-year medical students (the who) simple suturing and knot tying (the what). The degree of any skill competency can range from merely exposure to measured and demonstrated competency. The goal of the skill training could be to just perform the task once or to repeat the task until demonstrated proficiency. Returning to the example, let us postulate that the desired level of competence is limited skill exposure demonstrated by performing the task at least one time correctly. Agreeing that simulation can play a role, the level of fidelity is chosen. Can suturing and knot tying be taught using oranges or is the purchase of task trainers required? Any technical skill or procedure to be taught can be evaluated by asking these five questions.

At the University of Massachusetts Medical School, we have integrated simulation into the third-year medical student surgical rotation by developing surgical simulation scenarios. The objectives of the surgical simulation scenario

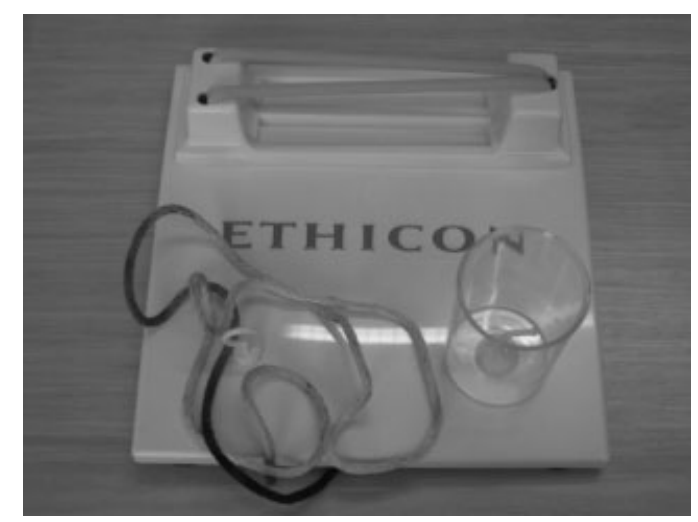

Figure 2 Task trainer for suturing and knot tying (Ethicon, Somerville, NJ). are to transfer knowledge of common surgical disorders, initiate psychomotor skill training for procedures related to disease management, and provide a venue for residents and students as educators. The format for these 2-hour sessions begins with a 45 -minute interactive case presentation, followed by three to five skill stations (10-20 minutes each), and concludes with a 15-minute wrap-up and evaluation. Surgical residents as well as other students can lead the case presentation and teach the skills. Three surgical simulation scenarios pertinent to colorectal surgeon educators are described.

The first scenario is entitled "Abdominal Pain." In this scenario, a general surgery resident leads an interactive discussion of a patient presenting with small bowel obstruction ( $\mathbf{- F i g . ~ 3 ) . ~ F o l l o w i n g ~ t h e ~ d i s c u s s i o n , ~ t h e ~ s m a l l ~ g r o u p ~ o f ~}$ students rotate to three skill stations each relevant to the management of a patient with small bowel obstruction: intravenous catheter insertion, nasogastric tube insertion, and placement of an indwelling urinary catheter in both the male and female pelvis (-Fig. 4). Task trainers are utilized. The degree of expected competence is initial exposure to the procedure.

A second scenario is designed to teach basic proctology and is entitled "Pain in the Butt." The session begins with a pretest consisting of 10 pictures of common anorectal conditions including a brief history of symptoms, followed by an interactive presentation by a colorectal surgeon, and finally five skill stations staffed by faculty and residents. The skill stations include (1) digital rectal examination and anoscopy, (2) fiberoptic flexible sigmoidoscopy, (3) rubber band ligation of internal hemorrhoid, (4) rectal prolapse reduction, and (5) incision and drainage of a pilonidal abscess (-Fig. 5). Simulation modalities include task trainers and manikins. The level of desired competency is initial exposure to the skills.

"Intestinal Stomas" is a surgical simulation scenario designed to teach not only about the types of intestinal stomas and rationale for their use, but also how to place and remove an ostomy appliance. This is effectively taught along with enterostomal nurses. This scenario is suitable for medical students and can also be tailored to nursing students and ostomy patients and their caregivers.

At our institution, we conduct surgical simulation scenarios entitled "Cold Blue Foot," "Breast Disease," and "Surgical Sepsis." All of these integrate hands-on skills training relevant to the topic. We are in the process of developing other themes appropriate to medical students involving neurosurgery, thoracic surgery, and plastic surgery.

\section{Mentorship Learning}

The mentorship model of surgical teaching ideally involves one-on-one, but possibly up to three students at a time. A mentor serves not only as a teacher and trainer, but also as an advisor and role model. We can all recall mentors who have significantly impacted our learning as well as our career choice. Most surgeons are comfortable with this model of learning and are experienced at mentoring to some degree. Given this, it is always valuable to reflect on one's mentoring style and look for ways to improve. 


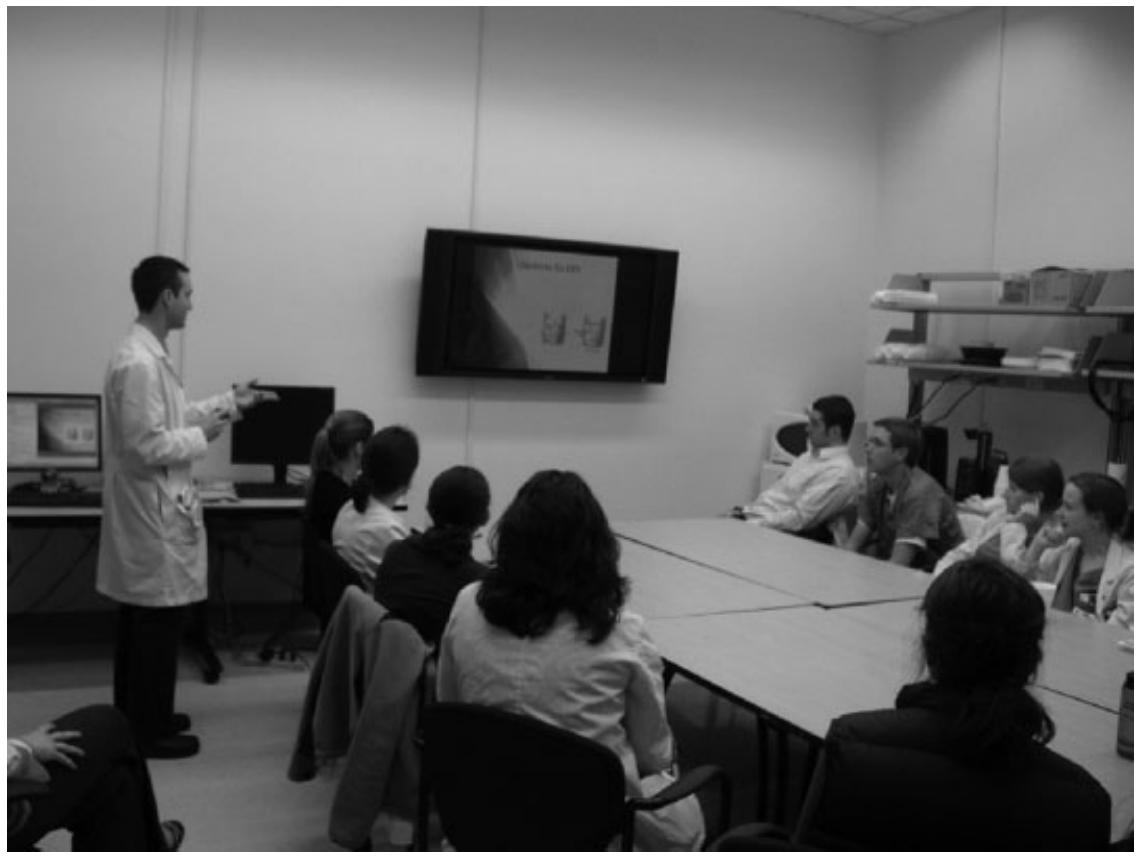

Figure 3 Small-student-group interactive case-based presentation.

Surgeons mentor students in the clinics or private offices, at the patient's bedside or on hospital rounds, and in the operating or procedure rooms. In all these settings, the surgeon has the opportunity to demonstrate not only higher-ordered thinking skills but also compassion and professionalism. After a period of modeling by the surgeon, allow the student to begin seeing the patient first. Although simply "shadowing" a surgeon has value, having the student first see the appropriate clinic patient has tremendous educational potential. When presenting the patient, the student needs to properly communicate the history to the faculty and begin to formulate a differential diagnosis. Although this method can
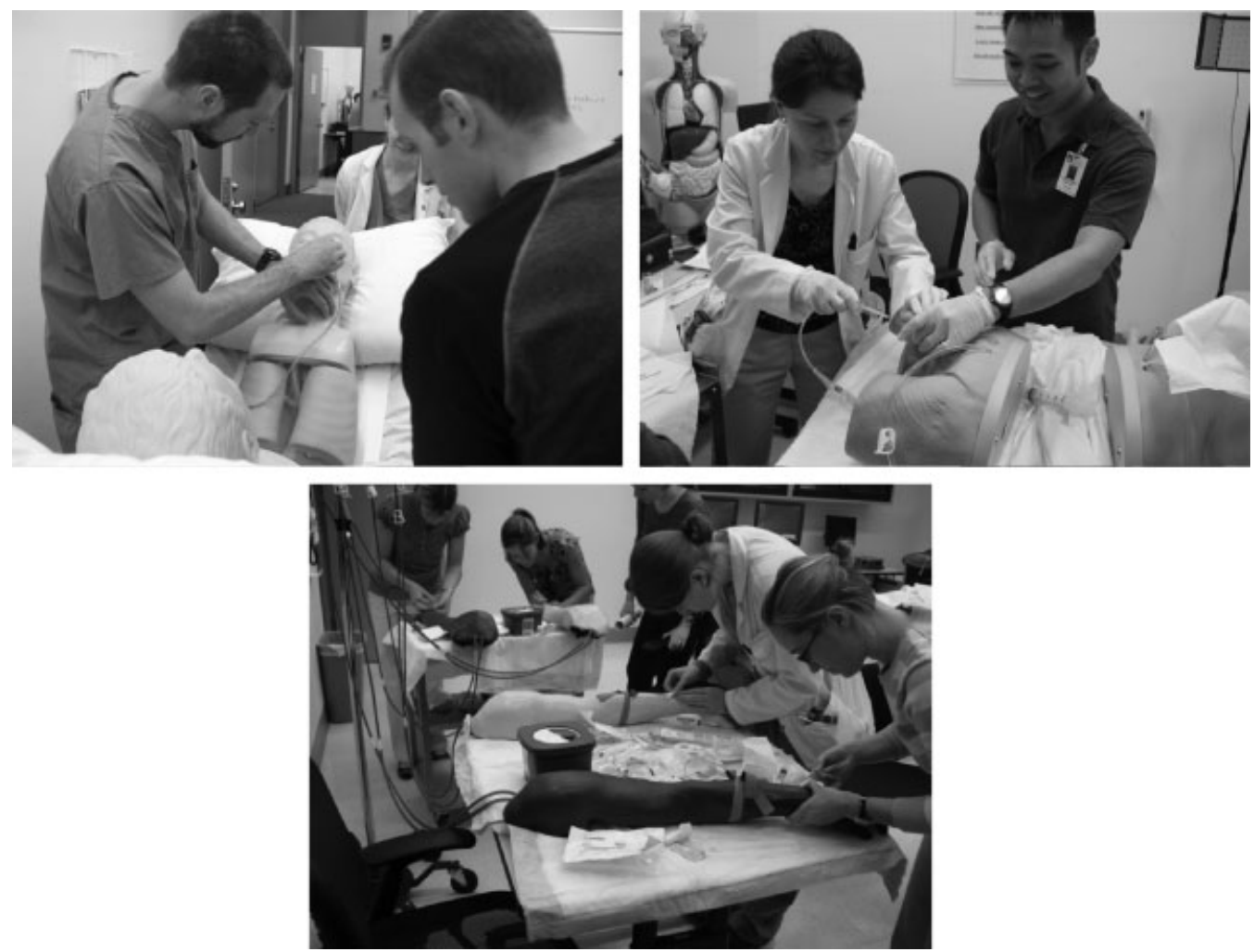

Figure 4 Surgical Simulation Scenario-Small Bowel Obstruction. Skills include nasogastric tube, Foley catheter, and intravenous line. 

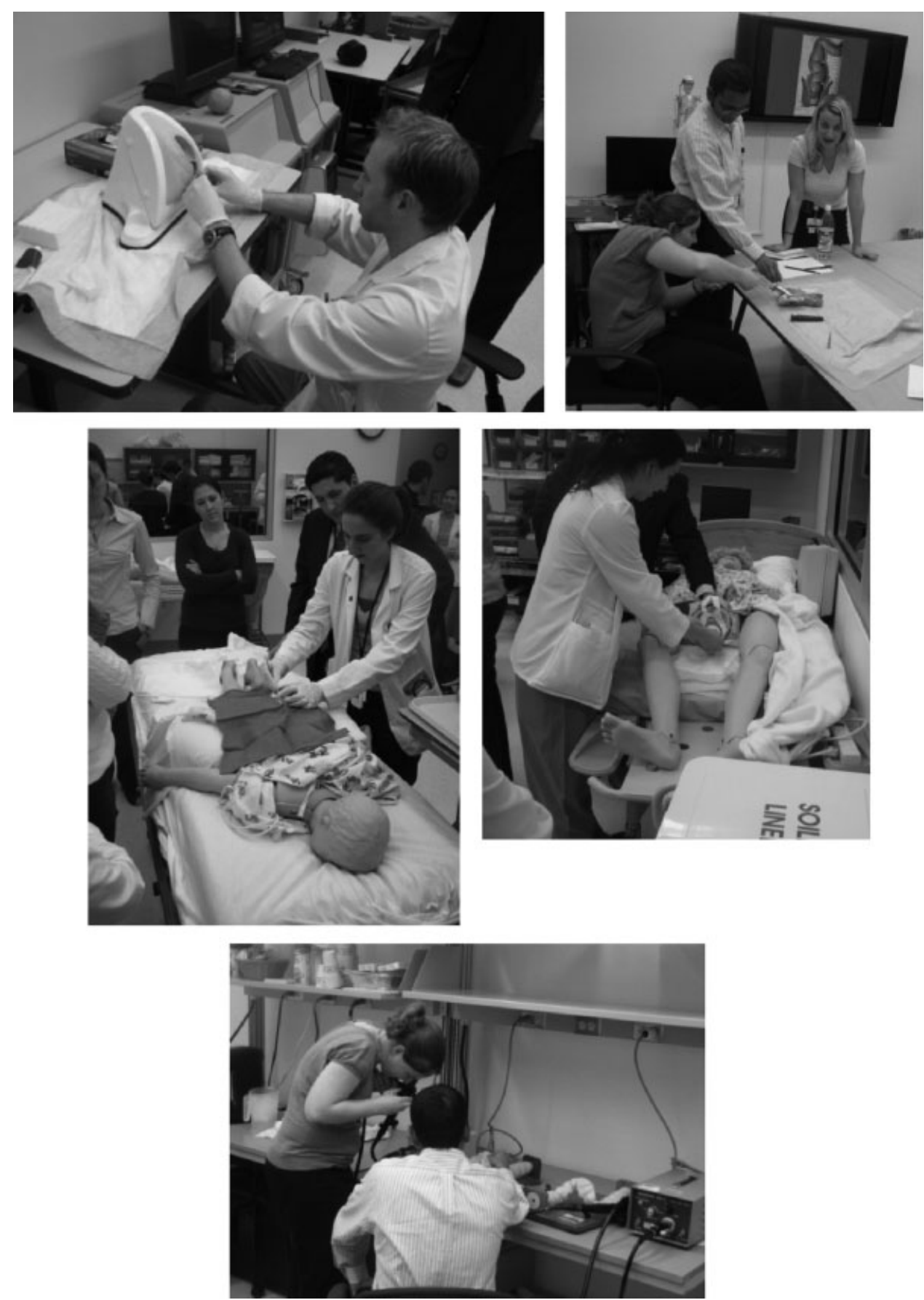

Figure 5 Surgical Simulation Scenario-Pain in the Butt. Skills include digital rectal examination and anoscopy, rubber band ligation of an internal hemorrhoid, incision and drainage of a pilonidal abscess, reduction of a rectal prolapse, and fiberoptic sigmoidoscopy.

take time during a busy clinic, with practice and selection of level appropriate specific patients the process can be smooth and rewarding for both student and educator.

Formal teaching rounds have become less frequent yet the student can learn a great deal about postoperative care when accompanying the staff surgeon on rounds. This is the opportunity for the surgeon educator to demonstrate superior "bedside manner" by the way a patient is listened to and cared for.

In the operating room, the student can be forgotten. Understandably, when the operative situation demands full concentration, the student's educational needs are deferred. This stated, the operating room is a perfect setting to teach about anatomy, disease processes, operative indications, potential complications, and medical devices. Make it a point to discuss the case as the patient is being positioned and prepped for the operation. Encourage the student to ask questions with the caveat that a lack of answer may only mean that the surgeon's concentration at the moment is elsewhere. Finally, a good time to review the operation with the student is at the completion when applying the dressings. Allowing the student to perform simple tasks such 
as suturing drains and placement of skin staples goes a long way toward making them feel part of the team.

Our mentees are requesting more involvement of surgeons in their medical school experience. ${ }^{9}$ Medical students suggest that surgeons give presentations in Years 1 and 2 of medical school, participate in surgery interest groups, and embrace small-group learning methods previously reviewed. These efforts can go a long way to not only promote our specialty, but also to ensure that those students not pursuing a surgical career will have proper knowledge about surgical diseases and surgical consultations.

\section{Conclusion}

As surgeons with typically no formal training in educational methods, we are responsible for teaching learners who are extremely bright, highly motivated, and immersed in a world of technology. We have the charge of teaching medical students surgical knowledge, decision making, and technical skills while simultaneously demonstrating professionalism and ethical principles. As clearly articulated by our mentees, the expectations are that the medical student can perform a surgical consultation, demonstrate preoperative counseling, understand basic operative procedures, and finally perform postoperative care with an awareness of potential surgical complications. ${ }^{9}$ These objectives can all be achieved by em- ploying traditional teaching methods as well as newer and creative modalities such as medical simulation. In addition to our role as educator, we must demonstrate the need and desire for life-long learning as we are forever students.

\section{References}

1 AAMC: DW: Applicant Matriculant File as of 10/19/2010. Available at: facts@aamc.org. Accessed March 30, 2012

2 Ferguson KJ. Facilitating student learning. In: Jeffries WB, Huggett $\mathrm{KN}$, eds. An Introduction to Medical Teaching. New York, NY: Springer; 2010:1-10

3 Kern DE, Thomas PA, Howard DM, Bass EB. Curriculum Development for Medical Education: A Six-Step Approach. Baltimore, MD: Johns Hopkins University Press; 1998

4 McKeachie WJ, Svinicki M. McKeachie's Teaching Tips. Strategies, Research, and Theory for College and University Teachers. Boston, MA: Houghton Mifflin; 2006

5 Med U. Available at: www.med-u.org. Accessed March 30, 2012

6 New England Journal of Medicine Videos in Clinical Medicine. Available at: www.nejm.org/multimedia/medical-videos. Accessed March 30, 2012

7 Procedures Consult. Available at: www.proceduresconsult.com. Accessed March 30, 2012

8 Medline Plus Surgical Procedures. Available at: www.nlm.nih.gov/ medlineplus/surgeryvideos. Accessed March 30, 2012

9 Fuller LT, Lin G, Matsui JY, Sobotka SA, Cooke DT. Teaching surgery to medical students: perspectives from our mentees. Bull Am Coll Surg 2008;93(7):48-53 